If the constants $a_{p q}$ are chosen so that the determinants $\Delta_{n}$ are all positive, $D_{n-2}(\lambda)$ and $D_{n}(\lambda)$ will have opposite signs when $D_{n-1}(\lambda)$ vanishes, and so the functions

$$
D(\lambda), \quad D_{1}(\lambda), \quad D_{2}(\lambda), \cdots, \quad D_{n}(\lambda)
$$

will form a Sturmian sequence.

It has been stated that the roots of the functions $\nabla_{n}(\lambda)$ in the Sturmian sequence separate one another. This is not always true for a Sturmian sequence when the functions are not polynomials, but it can be shown to be true in the present case, as follows. Let $g_{n}(s), g_{n}(t)$ be the cofactors of the constituents $f_{n}(t), f_{n}(s)$ in the determinant $F_{n}$; then from the properties of determinants

$$
F_{n-1} \cdot \Delta_{n}-g_{n}(s) g_{n}(t)=F_{n} \cdot \Delta_{n-1} .
$$

Dividing out by $\Delta_{n-1} \Delta_{n}$, we have

$$
h_{n}(s, t)=h_{n-1}(s, t)-\frac{g_{n}(s) g_{n}(t)}{\Delta_{n-1} \Delta_{n}} .
$$

We can now apply the theorem mentioned before to this equation and deduce that the roots of $h_{n-1}(s, t)$ are separated by those of $h_{n}(s, t)$, there being one root of $h_{n}(s, t)$ between each consecutive pair of roots of $h_{n-1}(s, t)$.

Bryn Mawr College, November, 1911.

\title{
ON THE CUBES OF DETERMINANTS OF THE SECOND, THIRD, AND HIGHER ORDERS.
}

BY PROFESSOR ROBERT E. MORITZ.

(Read before the San Francisco Section of the American Mathematical Society, April 8, 1911.)

While the square of a determinant of any order may be readily expressed as a determinant of the same order, I am not aware of the existence of a correspondingly simple method by means of which the cube of any determinant may be expressed in determinant form. For a determinant of the fourth order, $\Delta_{4}$, we have indeed from a well-known property of determinants

$$
\Delta_{4}{ }^{3} \equiv \Delta_{4}{ }^{\prime},
$$

where $\Delta_{4}^{\prime}$ is the determinant whose constituents are the co- 
factors of the corresponding constituents of $\Delta_{4}$. Another isolated case may be derived from the following general theorem due to Faà di Bruno:

The determinant which has for its first row the constituents

$$
x^{n}, \quad x^{n-1} y, \quad x^{n-2} y^{2}, \quad \cdots, \quad x y^{n-1}, \quad y^{n}
$$

and whose other rows are obtained by operating on the constituents of the first row successively by the symbolic operators

$$
\begin{gathered}
x^{\prime} \frac{d}{d x}+y^{\prime} \frac{d}{d y}, \frac{1}{1.2}\left(x^{\prime} \frac{d}{d x}+y^{\prime} \frac{d}{d y}\right)^{(2)}, \ldots, \\
\frac{1}{n !}\left(x^{\prime} \frac{d}{d x}+y^{\prime} \frac{d}{d y}\right)^{(n)}
\end{gathered}
$$

is a power, namely the $\frac{1}{2} n(n+1)$ th power, of the determinant $x y^{\prime}-x^{\prime} y$. For $n=2$, this theorem yields a determinant expression for $\left(x y^{\prime}-x^{\prime} y\right)^{3}$.

I

On multiplying each constituent of the determinant of the second order

by $\Delta_{2}$, we obtain

$$
\Delta_{2} \equiv\left|\begin{array}{cc}
a_{1} & a_{2} \\
b_{1} & b_{2}
\end{array}\right|
$$

$$
\Delta_{2}^{3} \equiv \Delta_{2}\left(a_{1} b_{2}-a_{2} b_{1}\right)^{2}=\left|\begin{array}{ll}
a_{1}\left(a_{1} b_{2}-a_{2} b_{1}\right) & a_{2}\left(a_{1} b_{2}-a_{2} b_{1}\right) \\
b_{1}\left(a_{1} b_{2}-a_{2} b_{1}\right) & b_{2}\left(a_{1} b_{2}-a_{2} b_{1}\right)
\end{array}\right|,
$$

and this in turn is equivalent to the bordered determinant

$$
\Delta_{2}^{3} \equiv \frac{1}{a_{1} b_{1}}\left|\begin{array}{ccc}
a_{1} b_{1} & a_{1} b_{2}-a_{2} b_{1} & a_{2} b_{2} \\
0 & a_{1}\left(a_{1} b_{2}-a_{2} b_{1}\right) & a_{2}\left(a_{1} b_{2}-a_{2} b_{1}\right) \\
0 & b_{1}\left(a_{1} b_{2}-a_{2} b_{1}\right) & b_{2}\left(a_{1} b_{2}-a_{2} b_{1}\right)
\end{array}\right| .
$$

Now subtract $a_{1}$ times the first row from the second, and add $b_{1}$ times the first row to the third, then

$$
\Delta_{2}^{3} \equiv \frac{1}{a_{1} b_{1}}\left|\begin{array}{rrr}
a_{1} b_{1} & a_{1} b_{2}-a_{2} b_{1} & a_{2} b_{2} \\
-a_{1}^{2} b_{1} & -2 a_{1} a_{2} b_{1} & -a_{2}{ }^{2} b_{1} \\
a_{1} b_{1}{ }^{2} & 2 a_{1} b_{1} b_{2} & a_{1} b_{2}{ }_{2}
\end{array}\right|,
$$


or, after interchanging the first and second rows and removing factors,

$$
\Delta_{2}^{3} \equiv\left|\begin{array}{ccc}
a_{1}^{2} & 2 a_{1} a_{2} & a_{2}^{2} \\
a_{1} b_{1} & a_{1} b_{2}+a_{2} b_{1} & a_{2} b_{2} \\
b_{1}^{2} & 2 b_{1} b_{2} & b_{2}^{2}
\end{array}\right|
$$

By adding the first and third columns to the second and putting

$$
a_{3}=a_{1}+a_{2}, \quad b_{3}=b_{1}+b_{2},
$$

we may write (1) in the form

$$
\Delta_{2}^{3} \equiv-\left|\begin{array}{ccc}
a_{1}^{2} & a_{2}^{2} & a_{3}^{2} \\
a_{1} b_{1} & a_{2} b_{2} & a_{3} b_{3} \\
b_{1}^{2} & b_{2}^{2} & b_{3}^{2}
\end{array}\right|
$$

We have then the following

Rule for the cube of any determinant of the second order:

Write down two lines of elements

$$
\begin{array}{lll}
a_{1}, & a_{2}, & a_{3}\left(=a_{1}+a_{2}\right), \\
b_{1}, & b_{2}, & b_{3}\left(=b_{1}+b_{2}\right),
\end{array}
$$

composed respectively of the constituents of the first row (or column) and their sum, the constituents of the second row (or column) and their sum, of any determinant of the second order. Its cube is the negative of the determinant of the third order the constituents of whose rows (or columns) are respectively the squares of the elements of the first line, the products of the elements of the first line by the corresponding elements of the second line, the squares of the elements of the second line.

II.

Next consider the determinant of the third order

$$
\Delta_{3} \equiv\left|\begin{array}{lll}
a_{1} & a_{2} & a_{3} \\
b_{1} & b_{2} & b_{3} \\
c_{1} & c_{2} & c_{3}
\end{array}\right| \equiv c_{1} c_{2} c_{3}\left|\begin{array}{ccc}
a_{1}^{\prime} & a_{2}^{\prime} & a_{3}^{\prime} \\
b_{1}^{\prime} & b_{2}^{\prime} & b_{3}^{\prime} \\
1 & 1 & 1
\end{array}\right|,
$$


where

$$
a_{1}^{\prime}=a_{1} / c_{1}, \quad a_{2}^{\prime}=a_{2} / c_{2}, \quad a_{3}{ }^{\prime}=a_{3} / c_{3}, \quad b_{1}{ }^{\prime}=b_{1} / c_{1}, \quad \text { etc. }
$$

Expressed as a determinant of the second order,

$$
\Delta_{3} \equiv c_{1} c_{2} c_{3}\left|\begin{array}{ll}
a_{2}{ }^{\prime}-a_{3}{ }^{\prime} & a_{3}{ }^{\prime}-a_{1}{ }^{\prime} \\
b_{2}{ }^{\prime}-b_{3}{ }^{\prime} & b_{3}{ }^{\prime}-b_{1}{ }^{\prime}
\end{array}\right|,
$$

from which by the use of (2)

where

$$
\Delta_{3}^{3} \equiv-\left(c_{1} c_{2} c_{3}\right)^{3}\left|\begin{array}{ccc}
x_{1}^{2} & x_{2}{ }^{2} & x_{3}^{2} \\
x_{1} y_{1} & x_{2} y_{2} & x_{3} y_{3} \\
y_{1}{ }^{2} & y_{2}{ }^{2} & y_{3}^{2}
\end{array}\right|,
$$

$$
\begin{aligned}
& x_{1}=a_{2}{ }^{\prime}-a_{3}{ }^{\prime}, \quad x_{2}=a_{3}{ }^{\prime}-a_{1}{ }^{\prime}, \quad x_{3}=a_{1}{ }^{\prime}-a_{2}{ }^{\prime}, \\
& y_{1}=b_{2}{ }^{\prime}-b_{3}{ }^{\prime}, \quad y_{2}=b_{3}{ }^{\prime}-b_{1}{ }^{\prime}, \quad y_{3}=b_{1}{ }^{\prime}-b_{2}{ }^{\prime} .
\end{aligned}
$$

But

$$
x_{1}=a_{2}{ }^{\prime}-a_{3}{ }^{\prime}=\frac{a_{2}}{c_{2}}-\frac{a_{3}}{c_{3}}=\frac{a_{2} c_{3}-a_{3} c_{2}}{c_{2} c_{3}}=\frac{-B_{1}}{c_{2} c_{3}},
$$

and similarly

$$
\begin{array}{r}
x_{2}=\frac{-B_{2}}{c_{3} c_{1}}, \quad x_{3}=\frac{-B_{3}}{c_{1} c_{2}}, \\
y_{1}=\frac{A_{1}}{c_{2} c_{3}}, \quad y_{2}=\frac{A_{2}}{c_{3} c_{1}}, \quad y_{3}=\frac{A_{3}}{c_{1} c_{2}},
\end{array}
$$

where the $A$ 's and $B$ 's are the cofactors of the corresponding constituents of $\Delta_{3}$. Substituting these values, we obtain after a slight reduction

$$
\Delta_{3}^{3} \equiv \frac{-1}{c_{1} c_{2} c_{3}}\left|\begin{array}{ccc}
A_{1}^{2} & A_{2}^{2} & A_{3}^{2} \\
A_{1} B_{1} & A_{2} B_{2} & A_{3} B_{3} \\
B_{1}^{2} & B_{2}^{2} & B_{3}^{2}
\end{array}\right| .
$$

A case of special interest is that in which the $c$ 's are unity. 
Thus

$$
\begin{aligned}
\left|\begin{array}{llr}
a_{1} & a_{2} & a_{3} \\
b_{1} & b_{2} & b_{3} \\
1 & 1 & 1
\end{array}\right|^{3} & \\
& \equiv-\left|\begin{array}{ccc}
\left(a_{2}-a_{3}\right)^{2} & \left(a_{3}-a_{1}\right)^{2} & \left(a_{1}-a_{2}\right)^{2} \\
\left(a_{2}-a_{3}\right)\left(b_{2}-b_{3}\right) & \left(a_{3}-a_{1}\right)\left(b_{3}-b_{1}\right) & \left(a_{1}-a_{2}\right)\left(b_{1}-b_{2}\right) \\
\left(b_{2}-b_{3}\right)^{2} & \left(b_{3}-b_{1}\right)^{2} & \left(b_{1}-b_{2}\right)^{2}
\end{array}\right| .
\end{aligned}
$$

III.

Before considering the cubes of determinants of orders higher than the third, we will establish the following

THeoRem I. Let $\Delta_{n} \equiv\left(a_{1} b_{2} \cdots n_{n}\right)$ represent any determinant of the nth order, and let $S$ denote the substitution which replaces

then

$$
\begin{aligned}
& a_{i} \text { by }\left(a_{i} k_{n+1}\right)=a_{i} k_{n+1}-a_{n+1} k_{i}, \\
& b_{i} \text { by }\left(b_{i} k_{n+1}\right)=b_{i} k_{n+1}-b_{n+1} k_{i}, \quad(i=12, \cdots, n) ; \\
& \cdot \cdot \cdot \cdot \cdot \cdot \cdot \cdot \cdot \cdot \cdot \cdot \cdot \cdot \\
& n_{i} \text { by }\left(n_{i} k_{n+1}\right)=n_{i} k_{n+1}-n_{n+1} k_{i}
\end{aligned}
$$

$$
S \Delta_{n}=k_{n+1}^{n-1} \Delta_{n+1}=k_{n+1}^{n-1}\left(a_{1} b_{2} \cdots n_{n} k_{n+1}\right),
$$

where $\Delta_{n+1}$ is the determinant of the $(n+1)$ th order, formed by bordering $\Delta_{n}$ on the right by

$$
a_{n+1}, \quad b_{n+1}, \cdots, \quad n_{n+1},
$$

and on the bottom by

$$
k_{1}, \quad k_{2}, \quad \cdots \quad k_{n}, \quad k_{n+1} .
$$

Proof.-Making the substitution indicated, we have to show that

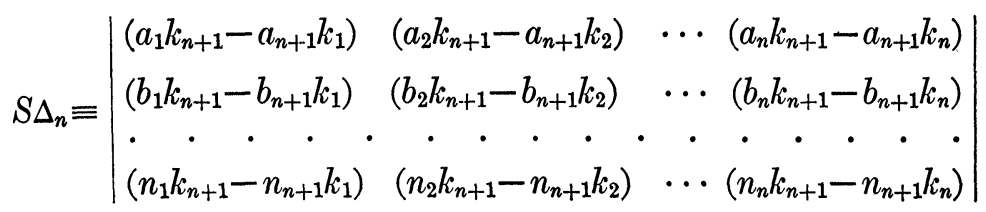




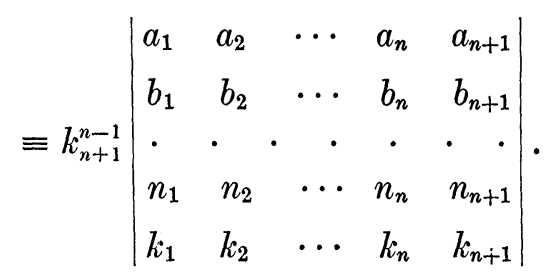

But this is merely the well-known identity which results from the reduction of any determinant of the $(n+1)$ th order to one of order $n$.

Definition.-Let us call the substitution $S$ which transforms any determinant $\Delta_{n} \equiv\left(a_{1} b_{2} \cdots n_{n}\right)$ into $\Delta_{n+1}=\left(a_{1} b_{2} \cdots n_{n} k_{n+1}\right)$ the substitution belonging to $\Delta_{n}$.

THEOREM II. If any cofactor $H_{i}^{(n)}$ belonging to $\Delta_{n}$ is transformed by the substitution belonging to $\Delta_{n}$, the result of the operation is the corresponding cofactor $H_{i}^{(n+1)}$ belonging to $\Delta_{n+1}$ multiplied by $k_{n+1}^{n-2}$.

This theorem is an immediate consequence of Theorem I, since the cofactor $H_{i}^{(n)}$ of $\Delta_{n}$, when bordered on the right by $a_{n+1}, b_{n+1}$, etc., and at the bottom by $k_{1}, k_{2}$, etc., becomes the cofactor $H_{i}^{(n+1)}$ of $\Delta_{n+1}$. Similar conclusions may be arrived at with regard to the transformation of the minors of any order.

Theorems I and II, though they appear here as immediate consequences of well-known results, enable us to derive from certain given forms and relations other forms and relations. For any identity between a determinant and its minors will, when operated on by the substitution belonging to the given determinant, give rise to another identity between a determinant of the next higher order and its minors. These theorems, therefore, permit us to proceed from relations holding for determinants of the second or third orders to corresponding relations between determinants of any order.

\section{IV.}

We now apply the theorems of the preceding section to each member of equation (3). $\Delta_{3}$ being of the third order, the substitution belonging to $\Delta_{3}$ is

$$
S=\left\{\begin{array}{ccc}
a_{i}, & b_{i}, & c_{i} \\
\left(a_{i} d_{4}\right), & \left(b_{i} d_{4}\right), & \left(c_{i} d_{4}\right)
\end{array}\right\} \quad(i=1,2,3),
$$


consequently

where

$$
S \Delta_{3}=d_{4}^{2} \Delta_{4}
$$

$$
\Delta_{4}=\left(a_{1} b_{2} c_{3} d_{4}\right) \text {, }
$$

$$
S A_{i}=d_{4} A_{i}^{(4)}, \quad S B_{i}=d_{4} B_{i}^{(4)}, \quad S C_{i}=d_{4} C_{i}^{(4)} \quad(i=1,2,3)
$$
where $A_{i}^{(4)}, B_{i}^{(4)}, C_{i}^{(4)}$, are the cofactors of the corresponding small letters in $\Delta_{4}$. Finally $S c_{i}=\left(c_{i} d_{4}\right)$. Hence

$$
S \Delta_{3}{ }^{3}=d_{4}{ }^{6} \Delta_{4}{ }^{3}=\frac{-d_{4}{ }^{6}}{\left(c_{1} d_{4}\right)\left(c_{2} d_{4}\right)\left(c_{3} d_{4}\right)}\left|\begin{array}{ccc}
A_{1}{ }^{(4)^{2}} & A_{2}{ }^{(4)^{2}} & A_{3}{ }^{(4)^{2}} \\
A_{1}{ }^{(4)} B_{1}{ }^{(4)} & A_{2}{ }^{(4)} B_{2}{ }^{(4)} & A_{3}{ }^{(4)} B_{3}{ }^{(4)} \\
B_{1}{ }^{(4)^{2}} & B_{2}{ }^{(4)^{2}} & B_{3}{ }^{(4)^{2}}
\end{array}\right|
$$

which, on removing the common factor $d_{4}{ }^{6}$ and dropping the indices, becomes

$$
\Delta_{4}^{3} \equiv \frac{-1}{\left(c_{1} d_{4}\right)\left(c_{2} d_{4}\right)\left(c_{3} d_{4}\right)}\left|\begin{array}{ccc}
A_{1}^{2} & A_{2}^{2} & A_{3}^{2} \\
A_{1} B_{1} & A_{2} B_{2} & A_{3} B_{3} \\
B_{1}{ }^{2} & B_{2}{ }^{2} & B_{3}{ }^{2}
\end{array}\right|
$$

\section{V.}

The general case now offers no further difficulty. The substitution belonging to $\Delta_{4}$ is

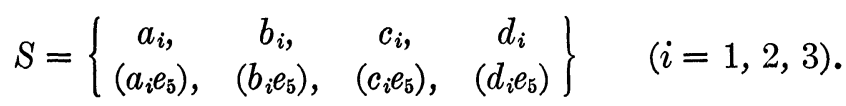

Operating on both members of (4) with $S$ we obtain

$$
\Delta_{5}^{3} \equiv \frac{-1}{\left(c_{1} d_{4} d_{5}\right)\left(c_{2} d_{4} e_{5}\right)\left(c_{3} d_{4} e_{5}\right)}\left|\begin{array}{ccc}
A_{1}^{2} & A_{2}{ }^{2} & A_{3}^{2} \\
A_{1} B_{1} & A_{2} B_{2} & A_{3} B_{3} \\
B_{1}{ }^{2} & B_{2}{ }^{2} & B_{3}{ }^{2}
\end{array}\right|,
$$

and hence by induction

(6) $\Delta_{n}{ }^{3} \equiv \frac{-1}{\left(c_{1} d_{4} \cdots n_{n}\right)\left(c_{2} d_{4} \cdots n_{n}\right)\left(c_{3} d_{4} \cdots n_{n}\right)}\left|\begin{array}{ccc}A_{1}{ }^{2} & A_{2}{ }^{2} & A_{3}{ }^{2} \\ A_{1} B_{1} & A_{2} B_{2} & A_{3} B_{3} \\ B_{1}{ }^{2} & B_{2}{ }^{2} & B_{3}{ }^{2}\end{array}\right|$, 
where the large letters are the cofactors of the corresponding small letters in $\Delta_{n}$. It will be noticed that each member of (6) is of degree $3 n$, as it should be.

UNIVERSITY OF WASHINGTON, March, 1911.

\section{NOTE ON THE MAXIMAL CYCLIC SUBGROUPS OF A GROUP OF ORDER $p^{m}$.}

BY PROFESSOR G. A. MILLER.

(Read before the San Francisco Section of the American Mathematical Society, October 28, 1911.)

IF $H$ is any non-invariant subgroup of a group $G$ of order $p^{m}$, $p$ being any prime number, it is well known that $H$ is transformed into itself by at least one of its conjugates under $G$ and hence by operators which are not contained in $H .^{*}$ If $H$ is cyclic and not contained in a larger cyclic subgroup of $G$, it is said to be a maximal cyclic subgroup of $G$. In what follows we shall establish the

TheOREM: A necessary and sufficient condition that every maximal cyclic subgroup of order $p^{\alpha}$ in a group $G$ of order $p^{m}, m>3$, is transformed into itself by no more than $p^{a+1}$ operators of $G$ is that $G$ contains one and only one cyclic subgroup of order $p^{m-1}$.

If we combine with this theorem some well-known properties of the groups of order $p^{m}$ which contain operators of order $p^{m-1}$, it results that there are only three non-cyclic groups of order $p^{m}$ which have the property that each of their maximal cyclic subgroups of order $p^{\alpha}$ is transformed into itself by only $p^{a+1}$ operators of the group. These three groups are the three non-cyclic groups of order $2^{m}$ which involve one and only one cyclic subgroup of order $2^{m-1}$.

To prove the theorem in question, we shall assume that $G$ does not involve any operator of order $p^{m-1}$, since the groups of order $p^{m}$ which contain operators of order $p^{m-1}$ are so well known. We shall also assume in what follows that $G$ satisfies the condition that each one of its maximal cyclic subgroups of order $p^{a}$ is transformed into itself by exactly $p^{a+1}$ operators of $G, p^{a}$ being the order of any one of the maximal cyclic subgroup of $G$.

* Cf. American Journal of Mathematics, vol. 23 (1901), p. 173. 